\title{
LOCALIZATION FOR FOURIER SERIES ON SU(2)
}

\author{
BY \\ R. MAYER( ${ }^{(1)}$
}

1. Introduction. Let $S U(2)$ be the group of $2 \times 2$ unitary matrices with determinant 1 , and for each positive integer $n$ let $\chi_{n}$ be the irreducible $n$-dimensional character of $S U(2),\left([6\right.$, pp. 151,163$]$ our character $\chi_{n}$ is Weyl's $\left.\chi_{n-1}\right)$. With each integrable function $f$ on $S U(2)$ there is associated a Fourier series

$$
f \sim \sum_{n=1}^{\infty} P_{n} f, \quad P_{n} f=f * n \chi_{n},
$$

where $*$ denotes convolution. For each $b \in S U(2)$ and each integer $k>0$ we set

$$
S_{k}(f: b)=\sum_{n=1}^{k} P_{n} f(b) \text {. }
$$

Let $N(b)$ be the space of functions $f \in L^{1}(S U(2))$ which vanish on a neighborhood $V_{f}$ of $b$. The main results of this paper are Theorems A-C below.

THEOREM A. Let $b \in S U(2), f \in N(b)$. If all of the first derivatives of $f$ (in the distribution sense) are functions in $L^{p}(S U(2))$ for some $p>3 / 2$, then $\lim _{n \rightarrow \infty} S_{n}(f: b)=0$. If $p<3 / 2$, there is a function $f \in N(b)$ whose first derivatives are all functions in $L^{p}(S U(2))$ and such that $\lim _{n \rightarrow \infty} S_{n}(f: b)$ does not exist.

THEOREM B. If $f \in N(b) \cap N(-b)$ and the first derivatives of $f$ are functions in $L^{1}(S U(2))$, then $\lim _{n \rightarrow \infty} S_{n}(f: b)=0$.

Say a function $f \in L^{1}(S U(2))$ is of bounded variation if its first derivatives are all measures.

THEOREM C. If $b \in S U(2)$ and $V \subset S U(2)$ is any nonvoid open set, then there is a function of bounded variation which vanishes on the complement of $V$ such that $\lim _{n \rightarrow \infty} S_{n}(f: b)$ does not exist.

The proof of Theorem $\mathrm{C}$ yields the following example. Let $e$ be the identity for $S U(2)$ and let

$$
\begin{aligned}
g(b) & =0 & & \text { if } \operatorname{tr}(b)<0, \\
& =\frac{1}{2} & & \text { if } \operatorname{tr}(b)=0, \\
& =1 & & \text { if } \operatorname{tr}(b)>0,
\end{aligned}
$$

Received by the editors September 26, 1966.

(1) Some of the results of this paper are contained in the author's Ph.D. Dissertation written at Columbia University under the direction of R. V. Kadison. The author would like to thank Professor Kadison for his helpful advice.

This research was supported by a National Science Foundation Cooperative Graduate Fellowship at Columbia University, and by the U.S. Army Research Office (Durham). 
where $\operatorname{tr}=$ trace. Then $\lim _{n \rightarrow \infty} S_{n}(g: b)=g(b)$ if $b \neq \pm e$, and $\lim _{n \rightarrow \infty} S_{n}(g: b)$ does not exist if $b= \pm e$. (Topologically $S U(2)$ is a 3 -sphere, $g=1$ on the northern hemisphere, $\frac{1}{2}$ on the equator, and 0 on the southern hemisphere, and the Fourier series for $f$ diverges at the north and south poles.)

2. Influence sets. Let $G$ be a compact group, let $S=\left\{S_{\alpha}\right\}(\alpha \in A)$ be a summation method for $G,[3, \S 5]$, let $M$ be a linear submanifold of $L^{1}(G)$, let $F$ be a closed subset of $G$ and let $b \in G$. We will say $F$ influences $S$ at $b$ for functions in $M$ if $\lim _{\alpha \in A} S_{\alpha}(f: b)=0$ for every $f \in M$ which vanishes on a neighborhood of $F$.

LEMMA 2.1. Let $G$ be a compact Lie group, and let $M$ be a left translation invariant linear submanifold of $L^{1}(G)$ which is also a module over $C^{\infty}(G)$ (i.e., fg $\in M$ for all $\left.f \in M, g \in C^{\infty}(G)\right)$. Let $S$ be a summation method for $G, b \in G$. Then there exists a unique closed set $I_{b}(S, M)$ in $G$ such that $I_{b}(S, M)$ influences $S$ at $b$ for functions in $M$ and $I_{b}(S, M)$ is contained in every closed set which influences $S$ at $b$ for functions in $M$. Also $I_{b}(S, M)=b I_{e}(S, M)$ where $e$ is the identity for $G$.

Proof. This was proved in [3, Theorem 5.7] for the case where $M=L^{1}(G)$. We will show that if $F_{1}$ and $F_{2}$ influence $S$ at $b$ for functions in $M$, then so does $F_{1} \cap F_{2}$. The rest of the proof is exactly like the proof given in [3]. Let $F_{1}, F_{2}$ influence $S$ at $b$ for functions in $M$ and let $f$ be a function in $M$ which vanishes on a neighborhood $U$ of $F_{1} \cap F_{2}$. Let $V_{1}, V_{2}$ be disjoint compact neighborhoods of $F_{1}-U, F_{2}-U$ respectively and let $h$ be a $C^{\infty}$ function on $G$ such that $h=1$ on $V_{1}$ and $h=0$ on $V_{2}$. Then $f h \in M$ and $f h$ vanishes near $F_{2}$ so $\lim _{\alpha \in A} S_{\alpha}(f h: b)=0$. Similarly $\lim _{\alpha \in A} S_{\alpha}(f(1-h): b)=0$, so $\lim _{\alpha \in A} S_{\alpha}(f: b)=0$ and $F_{1} \cap F_{2}$ influences $b$ for functions in $M$.

We will write $I_{e}(S, M)=I(S, M)$, and we say that the method $S$ has the localization property for functions in $M$ if $I(S, M)=\{e\}$. An element $b$ of $G$ is in $I(S, M)$ if and only if for each neighborhood $V$ of $b$ there is a function $f \in M$ such that $f$ vanishes on $G-V$ and $\lim _{\alpha \in A} S_{\alpha}(f: e) \neq 0$.

LEMmA 2.2. Let $G$ be a compact Lie group, let $S=\left\{S_{\alpha}\right\},(\alpha \in A)$ be a central summation method for $G[3, \S 5]$ and let $M$ be a linear submanifold of $L_{1}(G)$ which is left and right translation invariant and which is a module over $C^{\infty}(G)$. Then $a I(S, M) a^{-1}=I(S, M)$ for all $a \in G$.

Proof. Let $b \in I(S, M), a \in G$ and let $V$ be any neighborhood of $a b a^{-1}$. Then $a^{-1} V a$ is a neighborhood of $b$, so there is a function $f \in M$ such that $f$ vanishes on $G-a^{-1} V a$ and $\lim _{\alpha \in A} S_{\alpha}(f: e) \neq 0$. Define $g \in M$ by $g(x)=f\left(a^{-1} x a\right)$ for all $x \in G$. Then $g$ vanishes on $G-V$ and by [3, Equation (5.2)]

$$
\begin{aligned}
S_{\alpha}(g: e)=g * S_{\alpha}^{\curlyvee}(e) & =\int_{G} f\left(a^{-1} y a\right) S_{\alpha}^{\curlyvee}\left(y^{-1}\right) d y=\int_{G} f(y) S_{\alpha}^{\curlyvee}\left(a y^{-1} a^{-1}\right) d y . \\
& =\int_{G} f(y) S_{\alpha}^{\curlyvee}\left(y^{-1}\right) d y=f * S_{\alpha}^{\curlyvee}(e)=S_{\alpha}(f: e) .
\end{aligned}
$$

Thus $\lim _{\alpha \in A} S_{\alpha}(g: e) \neq 0$ and $a b a^{-1} \in I(S, M)$. 
Let $G$ be a compact Lie group and let $\&$ be the Lie algebra of $G$, i.e., the Lie algebra of left invariant vector fields on $G$. A function $f \in L^{1}(G)$ is of bounded variation if for each $D \in \mathbb{S}$ there is a constant $K_{D}$ such that

$$
|(D g, f)| \leqq K_{D}\|g\|_{\infty} \quad \text { for all } g \in C^{\infty}(G) \text {. }
$$

The space of functions of bounded variation on $G$ will be denoted by $B V$. For $1 \leqq p \leqq \infty$ let $W_{p}^{1}$ be the space of functions in $L^{1}(G)$ all of whose first derivatives are functions in $L^{p}(G)$. (A function $f \in L^{1}(G)$ is in $W_{p}^{1}$ if and only if for each $D \in$ (S) there is a function $D f \in L^{p}(G)$ such that $(D g, f)=-(g, D f)$, for all $g \in C^{\infty}(G)$.) It is routine to verify that $B V$ and $W_{p}^{1}$ are left and right translation invariant modules over $C^{\infty}(G)$.

3. Localization theorems. In this section $G$ will always denote $S U(2)$ and (S) will be the Lie algebra of $S U(2)$. For each integer $n \geqq 1$ let $E_{n}$ be the $n^{2}$ dimensional two-sided ideal in $L^{2}(G)$, let $\chi_{n}$ be the irreducible character in $E_{n}$ and let $P_{n}$ be the orthogonal projection onto $E_{n}$. Let $S=\left\{S_{n}\right\}(1 \leqq n<\infty)$, be the summation method for $G$ defined by

$$
S_{n}=P_{1}+\cdots+P_{n}
$$

In [3] it was shown that $I\left(S, L^{1}(G)\right)=G$. In this section we find $I\left(S, W_{p}^{1}\right)$ and $I(S, B V)$.

THEOREM 3.2. Let $S$ be the summation method defined in (3.1). Then

$$
\begin{aligned}
I\left(S: W_{p}^{1}\right) & =\{e\} \quad \text { if } p>3 / 2, \\
& =\{e\} \cup\{-e\} \quad \text { if } 1 \leqq p<3 / 2 .
\end{aligned}
$$

The proof will require a few lemmas.

Lemma 3.3. For any $D \in$ BS

$$
\begin{aligned}
\left(D \chi_{2}\right) n \chi_{n} & =D\left(\chi_{n+1}-\chi_{n-1}\right) \quad \text { for all } n \geqq 2 . \\
\left(D \chi_{2}\right) \sum_{k=1}^{n} k \chi_{k} & =D\left(\chi_{n}+\chi_{n+1}\right) \quad \text { for all } n \geqq 1 . \\
\left(D \chi_{n}\right)\left(3-\chi_{3}\right) & =\left((n+1) \chi_{n-1}-(n-1) \chi_{n+1}\right) \cdot D \chi_{2} \quad \text { for all } n \geqq 2 .
\end{aligned}
$$

Proof. We prove (3.4) by induction on $n$. Observe that (3.4) holds for $n=1$ if we set $\chi_{0}=0$. We will use the relations

$$
\begin{aligned}
\chi_{2} \chi_{k+1} & =\chi_{k}+\chi_{k+2}, \\
\chi_{3} \chi_{k} & =\chi_{k+2}+\chi_{k}+\chi_{k-2}, \quad(k \geqq 2)
\end{aligned}
$$

$[6$, p. 128]. For $n=2$ we have

$$
D \chi_{2} \cdot 2 \chi_{2}=D\left(\chi_{2}^{2}\right)=D\left(\chi_{3}+1\right)=D\left(\chi_{3}-1\right) .
$$


Now assume (3.4) for $n \leqq k$ where $k \geqq 2$. Applying the derivation $D$ to both sides of (3.7a) and using (3.4) for $n=k$ we get

$$
D \chi_{2} \cdot \chi_{k+1}+\chi_{2}\left(D \chi_{2} \cdot k \chi_{k}+D \chi_{k-1}\right)=D \chi_{k}+D \chi_{k+2} \text {. }
$$

Since

$$
\chi_{2} \cdot D \chi_{k-1}=D\left(\chi_{2} \cdot \chi_{k-1}\right)-D \chi_{2} \cdot \chi_{k-1}=D\left(\chi_{k}+\chi_{k-2}\right)-D \chi_{2} \cdot \chi_{k-1}
$$

we obtain

$$
(k+1) \chi_{k+1} \cdot D \chi_{2}+(k-1) \chi_{k-1} \cdot D \chi_{2}=D \chi_{k+2}-D \chi_{k-2} \text {. }
$$

If we use the induction hypothesis to evaluate $(k-1) \chi_{k-1} \cdot D_{\chi_{2}}$ we obtain (3.4) for $n=k+1$. (3.5) follows immediately from (3.4). Finally

$$
\begin{aligned}
\left((n+1) \chi_{n-1}-(n-1) \chi_{n+1}\right) D \chi_{2} & =\left((n-1) \chi_{n-1}-(n+1) \chi_{n+1}+2\left(\chi_{n-1}+\chi_{n+1}\right)\right) D \chi_{2} \\
& =D\left(\chi_{n}-\chi_{n-2}\right)-D\left(\chi_{n+2}-\chi_{n}\right)+2 \chi_{2} \chi_{n} D \chi_{2} \\
& =D\left(-\chi_{n+2}+2 \chi_{n}-\chi_{n-2}\right)+\chi_{n} D \chi_{2}^{2} \\
& =D\left(\left(3-\chi_{3}\right) \chi_{n}\right)-\chi_{n} D\left(3-\chi_{3}\right) \\
& =\left(3-\chi_{3}\right) D \chi_{n} .
\end{aligned}
$$

LEMMA 3.9. Let $h \in W_{p}^{1}$ where $p>3 / 2$. Then

$$
\lim _{n \rightarrow \infty} n \int_{G} h(a) \chi_{n}(a) d a=0 .
$$

Proof. Let $\Delta$ be the Laplace operator for $G, \Delta=D_{1}^{2}+D_{2}^{2}+D_{3}^{2}$ where $D_{1}, D_{2}, D_{3}$ is any basis for \&S which is orthonormal with respect to the Killing form for \&S. Each character $\chi_{n}$ is an eigenvector for $\Delta\left[1\right.$, p. 426]. Say $\Delta \chi_{n}=\lambda_{n} \chi_{n}$. Then it is well known that

$$
\lambda_{n}=\lambda_{2}\left(n^{2}-1\right) / 3 \text {. }
$$

((3.10) can be verified by induction using the relation $\lambda_{n}=\Delta \chi_{n}(e) / n$ together with (3.7a) and the fact that $D \chi_{n}(e)=0$ for all $n$ since $\chi_{n}$ has a maximum at $e$.) For $h \in W_{p}^{1}$ we have

$$
\begin{aligned}
\int_{G} h(a) \chi_{n}(a) d a & =3 \int_{G} h(a) \Delta \chi_{n}(a) d a / \lambda_{2}\left(n^{2}-1\right) \\
& =-3 \sum_{i=1}^{3}\left(D_{i} h, D_{i} \chi_{n}\right) / \lambda_{2}\left(n^{2}-1\right),
\end{aligned}
$$

so the lemma will follow if we show

$$
\lim _{n \rightarrow \infty}\left(g, D \chi_{n}\right) / n=0,
$$

for all $g \in L^{p}(G), D \in \mathbb{B S}$. Condition (3.11) is satisfied for any $g$ in the representative ring of $G$, and since the representative ring is dense in $L^{p}(G)$ for $1 \leqq p<\infty$ it follows from [3, Lemma 5.10] that (3.11) holds for all $g \in L^{p}(G)(3 / 2<p<\infty)$ if and only if

$$
\left\{n^{-1}\left\|D_{\chi_{n}}\right\|_{q}: 1 \leqq n<\infty\right\}
$$

is bounded for each $q, 1<q<3$. 
Now $\Delta \chi_{2}^{2}=2 \chi_{2} \Delta \chi_{2}+2 \sum_{i=1}^{3}\left(D_{i} \chi_{2}\right)^{2}$ so using (3.10) we get

$$
\sum_{i=1}^{3}\left(D_{i} \chi_{2}\right)^{2}=\left(\chi_{3}-3\right) \lambda_{2} / 3
$$

Since $D_{i} \chi_{2}$ is real it follows that

$$
\left(3-\chi_{3}\right)^{-1 / 2} D \chi_{2} \in L^{\infty}(G), \quad \text { for all } D \in \text { SS. }
$$

By (3.6) and (3.14) we see that there is a constant $K_{D}$ such that

$$
\begin{aligned}
\left\|D \chi_{n}\right\|_{q} & =\left\|\left((n+1) \chi_{n-1}-(n-1) \chi_{n+1}\right) D \chi_{2} \cdot\left(3-\chi_{3}\right)^{-1}\right\|_{q} \\
& \leqq K_{D}\left\|\left((n+1) \chi_{n-1}-(n-1) \chi_{n+1}\right)\left(3-\chi_{3}\right)^{-1 / 2}\right\|_{q}
\end{aligned}
$$

and hence

$$
n^{-1}\left\|D \chi_{n}\right\|_{q} \leqq K_{D}\left\|\chi_{n-1}-\chi_{n+1}+n^{-1} \chi_{2} \chi_{n}\right\|_{\infty}\left\|\left(3-\chi_{3}\right)^{-1 / 2}\right\|_{q} .
$$

Using the explicit formulas for the characters and Haar measure on $S U(2)$ discussed in $[6$, pp. 151,163$]$ we get $\chi_{n+1}(a)-\chi_{n-1}(a)=\varepsilon^{n}+\varepsilon^{-n}$ where the eigenvalues of $a$. are $\varepsilon, \varepsilon^{-1}$, so

$$
\left\|\chi_{n-1}-\chi_{n+1}+n^{-1} \chi_{2} \chi_{n}\right\|_{\infty} \leqq 4
$$

and

$$
\left\|\left(3-\chi_{3}\right)^{-1 / 2}\right\|_{q}^{q}=2^{1-q} \cdot \frac{1}{\pi} \int_{0}^{\pi} \sin ^{2-q}(t) d t .
$$

(3.12) thus follows from (3.15), and the proof of Lemma 3.9 is complete. (Note that $W_{p}^{1} \supset W_{\infty}^{1}$ for $1 \leqq p \leqq \infty$.)

Let $f \in W_{p}^{1}$ where $p>3 / 2$ and suppose $f$ vanishes on a neighborhood $V$ of $e$. By [3, Equation 5.12] we have

$$
S_{n}(f: e)=f * S_{n}^{\vee}(e)=\int_{G} f(a)\left((n+1) \chi_{n}(a)-n \chi_{n+1}(a)\right)\left(2-\chi_{2}(a)\right)^{-1} d a .
$$

Since $f$ vanishes near $e$ and $\left(2-\chi_{2}\right)^{-1}$ is infinitely differentiable except at $e$ it follows that $h=\left(2-\chi_{2}\right)^{-1} f$ is a function in $W_{p}^{1}$ which vanishes near $e$, and

$$
S_{n}(f: e)=\int_{G} h(a)\left((n+1) \chi_{n}(a)-n \chi_{n+1}(a)\right) d a .
$$

By Lemma $3.9 \lim _{n \rightarrow \infty} S_{n}(f: e)=0$, hence $I\left(S, W_{p}^{1}\right)=\{e\}$ if $p>3 / 2$.

LEMMA 3.16. Let $f$ be a function in $L^{2}(S U(2))$ which is continuously differentiable except at a single point $a$, and let $D \in \mathbb{S}$. Suppose that the pointwise derivative $D f$ (which is defined except at a) is a function in $L^{1}$. Then the derivative of $f$ considered as a distribution is the function $D f$.

(The proof is a standard kind of argument, and is omitted.)

Lemma 3.17. Suppose $1 \leqq p<3 / 2$. Then there exists a function $f \in W_{p}^{1}$ which vanishes on a neighborhood of e such that $\lim _{n \rightarrow \infty} S_{n}(f: e)$ does not exist. 
Proof. Let $F$ be the function on $[0, \pi)$ defined by

$$
\begin{aligned}
F(t) & =0 \quad \text { if } 0 \leqq t \leqq \pi / 2 \\
& =\csc t-1 \quad \text { if } \pi / 2 \leqq t<\pi .
\end{aligned}
$$

Let $\theta$ be the function on $G$ defined by

$$
\theta=\arccos \left(\frac{1}{2} \chi_{2}\right) .
$$

Let $f=F \circ \theta$. Then $f \in L^{2}(G) \subset L^{p}(G),[6, \mathrm{p} .163]$, and for any $D \in \mathbb{S}$ we have

$$
\begin{aligned}
D f(a) & =0 \quad \text { if } 0 \leqq \theta(a) \leqq \pi / 2 \\
& =-\csc \theta(a) \cot \theta(a) D \theta(a) \quad \text { if } \pi / 2 \leqq \theta(a)<\pi .
\end{aligned}
$$

Now

$$
D \theta=-\frac{1}{2}\left(1-\left(\frac{1}{2} \chi_{2}\right)^{2}\right)^{-1 / 2} D \chi_{2}=-\left(3-\chi_{3}\right)^{-1 / 2} D \chi_{2}
$$

is bounded by (3.14), so $D f \in L^{1}(G)$, and by Lemma 3.16 the distribution derivative of $f$ coincides with the function $D f$. Since $D f \in L^{p}(G)$ for $1 \leqq p<3 / 2$, we have $f \in W_{p}^{1}$ for $1 \leqq p<3 / 2$.

$$
P_{k} f=\left(f, \chi_{k}\right) \cdot \chi_{k}=2 \chi_{k} / \pi k, \quad k \text { odd, } k>1 .
$$

Hence $P_{k} f(e)$ does not tend to 0 as $k$ becomes large and hence $\lim _{n \rightarrow \infty} S_{n}(f: e)$ $=\lim _{n \rightarrow \infty} \sum_{1}^{n} P_{k} f(e)$ does not exist.

LEMMA 3.21. Let $f \in L^{1}(G)$ and suppose that $f$ vanishes on a neighbor hood $V$ of $\{e\} \cup\{-e\}$. Then

$$
\lim _{n \rightarrow \infty} \int_{G} f(a) \chi_{n}(a) d a=0 .
$$

Proof. Let $L^{p}(G-V)$ be the subspace of $L^{p}(G)$ consisting of those functions in $L^{p}(G)$ which vanish on $V$. Since $\left\{\chi_{n}\right\}$ is an orthonormal set in $L^{2}(G)$, (3.22) holds for all $f \in L^{2}(G-V)$ and since $L^{2}(G-V)$ is dense in $L^{1}(G-V)$ it follows from [3, Lemma 5.10] that (3.22) holds for all $f \in L^{1}(G-V)$ if and only if the set of numbers $\left\{\sup \left\{\left|\chi_{n}(a)\right|: a \in G-V\right\}: 1 \leqq n<\infty\right\}$ is bounded. It is easy to verify that this is the case.

LEMMA 3.23. $I\left(S: W_{1}^{1}\right)=\{e\} \cup\{-e\}$.

Proof. Let $f$ be a function in $W_{1}^{1}$ which vanishes on a neighborhood $V$ of $\{e\} \cup\{-e\}$. Then by (3.5)

$$
S_{n}(f: e)=\int_{G} f(a)\left(\sum_{k=1}^{n} k \chi_{k}(a)\right) d a=\int_{G}\left(f(a) / D \chi_{2}(a)\right) \cdot D\left(\chi_{n}+\chi_{n+1}\right)(a) d a
$$

for any $D \in \mathbb{S}, D \neq 0$. Let $D_{1}, D_{2}, D_{3}$ be an orthonormal basis for \&S with respect to the Killing form for $\mathcal{S}$, and let $F_{j}=\left\{a \in G: D_{j} \chi_{2}(a)=0\right\}(1 \leqq j \leqq 3)$. It follows from (3.13) and the fact that $\chi_{3}(a)=3$ if and only if $a= \pm e$ that $F_{1} \cap F_{2} \cap F_{3}$ 
$=\{e\} \cup\{-e\}$. Let $U$ be a neighborhood of $\{e\} \cup\{-e\}$ such that $U^{-} \subset V$ and let $F_{j}^{U}=F_{j} \cap(G-U)$ for $1 \leqq j \leqq 3$. Then each $F_{j}^{U}$ is compact and

$$
\bigcap_{j=1}^{3} F_{j}^{U}=\varnothing \text {. }
$$

Choose open sets $W_{j}(1 \leqq j \leqq 3)$ in $G$ so that

$$
W_{j} \supset F_{j}^{U} \text { and } \bigcap_{j=1}^{3} W_{j}^{-}=\varnothing .
$$

Let $\left(W_{j}^{-}\right)^{\prime}$ be the complement of $W_{j}^{-}$so that $\left\{\left(W_{j}^{-}\right)^{\prime}: 1 \leqq j \leqq 3\right\}$ is an open cover for $S U(2)$. Let $\left\{g_{1}, g_{2}, g_{3}\right\}$ be a $C^{\infty}$ partition of unity for $G$ subordinate to this cover (so $g_{j}=0$ on $W_{j}^{-}$) and let $h$ be a $C^{\infty}$ function such that $h=0$ on $U$ and $h=1$ on $G-V$. Let $\lambda_{j}=g_{j} h$ for $1 \leqq j \leqq 3$. Then we have

$$
f=\sum_{j=1}^{3} \lambda_{j} f
$$

Moreover each $\lambda_{j} / D_{j} \chi_{2}$ is a $C^{\infty}$ function since $\lambda_{j}$ vanishes on a neighborhood of the zeros of $D_{\chi_{2}}$. By (3.24)

$$
S_{n}(f: e)=\sum_{j=1}^{3} S_{n}\left(\lambda_{j} f: e\right)=\sum_{j=1}^{3} \int_{G}\left(\lambda_{j} f / D_{j} \chi_{2}\right) \cdot D_{j}\left(\chi_{n+1}+\chi_{n}\right) d \mu .
$$

Each function $\lambda_{j} f / D_{j} \chi_{2}$ is in $W_{1}^{1}$, since $W_{1}^{1}$ is a module over $C^{\infty}(G)$. Hence

$$
S_{n}(f: e)=-\sum_{j=1}^{3} \int_{G} D_{j}\left(\lambda_{j} f / D_{j} \chi_{2}\right) \cdot\left(\chi_{n+1}+\chi_{n}\right) d \mu
$$

and it follows from Lemma 3.21 that $\lim _{n \rightarrow \infty} S_{n}(f: e)=0$. Hence $I\left(S, W_{1}^{1}\right)$ $\subset\{e\} \cup\{-e\}$. By Lemma $3.17 I\left(S, W_{1}^{1}\right)$ contains $\{e\}$ as a proper subset so Lemma 3.23 follows.

We have already observed that $I\left(S, W_{p}^{1}\right)=\{e\}$ if $p>3 / 2$. The rest of Theorem 3.2 is clear from Lemma 3.17 and Lemma 3.23. If $f \in L^{1}(G)$ and $f$ vanishes near $e$ then either $\lim _{n \rightarrow \infty} S_{n}(f: e)=0$ or $\lim _{n \rightarrow \infty} S_{n}(f: e)$ does not exist [3, Theorem 7.12]. Hence Theorems A and B of the introduction follow from Theorem 3.2.

TheOREM 3.25. $I(S, B V)=G$. (This is Theorem $\mathrm{C}$ of the introduction.)

Again the proof requires a few lemmas.

LEMMA 3.26. Let $f$ be a class function in $L^{1}(S U(2))$ and let $f^{\prime}$ be the even function on $[-\pi, \pi]$ defined by

$$
f^{\prime}(t)=f\left(x_{t}\right) \quad \text { where } x_{t}=\operatorname{diag}\left(e^{i t}, e^{-i t}\right) .
$$

Suppose that $f^{\prime} \in L^{1}(-\pi, \pi)$ and let $f^{\prime} \sim \sum C_{n} e^{\text {int }}$ be the Fourier series for $f^{\prime}$. Then

$$
S_{n}(f: a)=\sum_{-n+1}^{n-1} C_{k} e^{i k \theta(a)}-\left(C_{n} \chi_{n-1}(a)+C_{n+1} \chi_{n}(a)\right)
$$

for all $a \in S U(2), n=1,2, \ldots,(\theta$ is as in (3.19)). 
Proof. $C_{n}=C_{-n}$ so for any $n>1$

$$
\int_{G} f(a) \chi_{n}(a) d a=\frac{1}{\pi} \int_{-\pi}^{\pi} f^{\prime}(t) \sin n t \sin t d t=C_{n-1}-C_{n+1} .
$$

Using the relation $\chi_{n}=2 \cos (n-1) \theta+\chi_{n-2}$, for $n \geqq 2,\left(\chi_{0}=0\right)$ we get

$$
P_{n} f=\left(f, \chi_{n}\right) \chi_{n}=2 C_{n-1} \cos (n-1) \theta+C_{n-1} \chi_{n-2}-C_{n+1} \chi_{n}
$$

for $n \geqq 2$. Since $P_{1} f=C_{0}-C_{2}$ and

$$
S_{n}(f: a)=\sum_{k=1}^{n} P_{k} f(a)
$$

(3.27) follows from (3.29).

LEMMA 3.30. For each $t \in(-2,2)$ let $\lambda_{t}$ be the function on $G$ defined by

$$
\begin{aligned}
\lambda_{t}(a) & =0 & & \text { if } \chi_{2}(a)<t, \\
& =\frac{1}{2} & & \text { if } \chi_{2}(a)=t, \\
& =1 & & \text { if } \chi_{2}(a)>t .
\end{aligned}
$$

Then the Fourier series for $\lambda_{t}$ diverges at $\pm e$ and converges elsewhere.

Proof. $\lambda_{t}^{\prime}$ is clearly in $L^{1}(-\pi, \pi)$ and a straightforward calculation shows that

$$
\lambda_{t}^{\prime}(s) \sim \sum C_{n} e^{i n s}
$$

where $C_{n}=\sin \left(n \arccos \left(\frac{1}{2} t\right)\right) / \pi n$. We will write $T=\arccos \left(\frac{1}{2} t\right)$. By (3.27)

$$
S_{n}\left(\lambda_{t}: a\right)=\sum_{-n+1}^{n-1} \frac{\sin k T}{\pi k} e^{i k \theta(a)}-\frac{\sin n T}{\pi n} \chi_{n-1}(a)-\frac{\sin (n+1) T}{\pi(n+1)} \chi_{n}(a) .
$$

We know that

$$
\lim _{n \rightarrow \infty} \sum_{-n}^{n} \frac{\sin k T}{\pi k} e^{i k \theta(a)}=\lambda_{t}^{\prime}(\theta(a))
$$

for all $\theta(a)$, so $\lim _{n \rightarrow \infty} S_{n}\left(\lambda_{t}: a\right)$ exists if and only if the limit

$$
\lim _{n \rightarrow \infty}(\sin n T) \chi_{n-1}(a) / n+(\sin (n+1) T) \chi_{n}(a) /(n+1)
$$

exists. If $a \neq \pm e$ this limit is clearly 0 . If $a=e$ (3.33) becomes

$$
\lim _{n \rightarrow \infty}(n-1)(\sin n T) / n+n(\sin (n+1) T) /(n+1)
$$

and this limit does not exist for $T \in(0, \pi)$. Similarly $\lim _{n \rightarrow \infty} S_{n}\left(\lambda_{t}:-e\right)$ does not exist.

In $\$ 4$ we will show that the functions $\lambda_{t} \in B V$ by computing their derivatives explicitly. (The explicit formula for the derivatives will be used in a later paper.) For the present we assume $\lambda_{t} \in B V$. 
Proof of Theorem 3.25. Every conjugacy class of $S U(2)$ is of the form

$$
K_{t}=\left\{a \in S U(2): \chi_{2}(a)=t\right\} \quad \text { for some } t \in[-2,2] .
$$

Any neighborhood $N$ of $K_{t}$ contains a set of the form

$$
N_{t \varepsilon}=\left\{a \in G:\left|\chi_{2}(a)-t\right|<\varepsilon\right\} .
$$

Let $f_{t \varepsilon}$ be a continuously differentiable class function on $G$ such that

$$
\begin{aligned}
f_{t \varepsilon}(a) & =0 & & \text { if } \chi_{2}(a) \leqq t-\varepsilon, \\
& =-1 & & \text { if } \chi_{2}(a) \geqq t+\varepsilon,
\end{aligned}
$$

and such that $f_{t \varepsilon}=0$ near $-e$ and $f_{t \varepsilon}=-1$ near $e$. Then $f_{t \varepsilon}^{\prime} \sim \sum C_{n} e^{i n s}$ where $C_{n}=o\left(n^{-1}\right)$ and it follows from (3.27) that $f_{t \varepsilon}$ has an everywhere convergent Fourier series. Let $t \in(-2,2)$, let $N$ be any neighborhood of $K_{t}$, and let $N_{t \varepsilon} \subset N$. Then $\lambda_{t}+f_{t \varepsilon} \in B V, \lambda_{t}+f_{t \varepsilon}$ vanishes on the complement of $N$ and $\lim _{n \rightarrow \infty} S_{n}\left(\lambda_{t}+f_{t \varepsilon}: e\right)$ $=\lim _{n \rightarrow \infty} S_{n}\left(\lambda_{t}: e\right)$ does not exist. Hence $N \cap I(S, B V) \neq \varnothing$ for every neighborhood $N$ of $K_{t}$, hence $K_{t} \cap I(S, B V) \neq \varnothing$ and by Lemma 2.2, $K_{t} \subset I(S, B V)$. Clearly $K_{t} \subset I(S, B V)$ for $t= \pm 2$ and it follows that $I(S, B V)=G$.

4. Calculation of some derivatives. Let $G$ be a compact Lie group with Lie algebra (S). For each $D \in$ SS let $D_{R}$ be the right invariant vector field on $G$ defined by $D_{R}=-J D J$ where $(J f)(x)=\bar{f}\left(x^{-1}\right)=f^{*}(x)$ for any function $f$ on $G$.

LEMMA 4.1. Let $G$ be a compact connected semisimple Lie group with Lie algebra (S), let $\mathfrak{A}$ be an abelian subalgebra of $\mathbb{S}$, and let $E$ be a minimal two-sided ideal in the convolution algebra $L^{2}(G)$, of dimension $d_{E}^{2}$. Then there exists an irreducible matrix representation $a \rightarrow\left(\mu_{k j}(a)\right)$ of $G$ and a family $\left\{\phi_{k}\right\}\left(1 \leqq k \leqq d_{E}\right)$ of real linear functionals on (s) such that

$$
\begin{aligned}
& \mu_{k j} \in E, \quad 1 \leqq k, j \leqq d_{E}, \\
& D \mu_{k j}=i \phi_{j}(D) \mu_{k j}, \quad 1 \leqq k, j \leqq d_{E}, \quad D \in \mathfrak{A}, \\
& D_{R} \mu_{k j}=i \phi_{k}(D) \mu_{k j}, \quad 1 \leqq k, j \leqq d_{E}, \quad D \in \mathfrak{A} \text {. }
\end{aligned}
$$

Proof. The space $E$ is translation invariant, and hence is invariant under each operator $D \in$ SS. Also each restriction $\left.D\right|_{E}, D \in B S$ is skew Hermitian since $(D f, g)$ $=-(f, D g)$ for all $f, g \in E$. The complex associative algebra generated by $\left\{\left.D\right|_{E}: D \in \mathfrak{A}\right\}$ is an abelian selfadjoint algebra of operators on $E$, and hence is generated by a single operator $\left.T\right|_{E}$. Using left invariance of $T$ it is easy to verify that $T(f * g)=f * T g$ for all $f, g \in E$, and from this it follows that any eigenspace of $\left.T\right|_{E}$ is a left ideal in $E$. Since $E$ is the orthogonal sum of the eigenspaces of $\left.T\right|_{E}$ and any left ideal in $E$ is the orthogonal sum of minimal left ideals we can write $E=\sum E_{k}\left(1 \leqq k \leqq d_{E}\right)$ where each $E_{i}$ is a minimal left ideal invariant under $T$ (and hence under $\mathfrak{A}$ ), and the ideals $E_{k}$ are mutually orthogonal. Write $\mathfrak{A}_{R}=\left\{D_{R}: D \in \mathfrak{Q}\right\}$. Each ideal $E_{k}$ is invariant under $\mathfrak{A}_{R}\left(\left[4\right.\right.$, p. 294] and note that $D_{R} P_{E}$ is a bounded 
right invariant operator where $P_{E}$ is the projection onto $E$ ). Write $E_{k}=L^{2}(G) * f_{k}$ where $f_{k}=f_{k}^{*}$ is a minimal idempotent in $L^{2}(G)$, and let $F_{k}=J\left(E_{k}\right)=f_{k} * L^{2}(G)$. Then $F_{k} \subset E$ and each $F_{k}$ is a minimal right ideal which is invariant under $\mathfrak{A}$ and $\mathfrak{A}_{R} . E_{k} \cap F_{j}=f_{j} * L^{2}(G) * f_{k}=f_{j} * E * f_{k}$ is a 1-dimensional subspace of $E[2, \mathrm{p}$. 104], and the spaces $E_{k} \cap F_{j}$ are mutually orthogonal and invariant under $\mathfrak{A}$ and $\mathfrak{A}_{R}$. For each $D \in \mathfrak{A}$ and $1 \leqq k \leqq d_{E}$ let $\phi_{k}(D)$ be the number such that $\left.D\right|_{E_{k}}=i \phi_{k}(D) I_{k}$, where $I_{k}$ is the identity operator on $E_{k} .\left.D\right|_{E_{k}}$ is a scalar by Schur's lemma and $\phi_{k}(D)$ is real because $\left.D\right|_{E}$ is skew Hermitian. If $f \in F_{k}$ and $D \in \mathfrak{A}$ then $D_{R} f=-J D J f$ $=-J\left(i \phi_{k}(D) J f\right)=i \phi_{k}(D) f$, and hence if $\mu \in E_{k} \cap F_{j}$ we have

$$
D \mu=i \phi_{k}(D) \mu, \quad D_{R} \mu=i \phi_{j}(D) \mu .
$$

Let $f_{k 1}\left(1 \leqq k \leqq d_{E}\right)$ be a unit vector in $E_{1} \cap F_{k}$. Then $\left(f_{k 1}\right)^{-}\left(1 \leqq k \leqq d_{E}\right)$ is an orthonormal basis for $\bar{E}_{1}$. The matrix coordinates of the left regular representation $L$ restricted to $\bar{E}_{1}$ relative to the basis $\left(f_{k 1}\right)^{-}$are given by $\mu_{k j}(a)=\left(L(a) \bar{f}_{j 1}, \bar{f}_{k 1}\right)$ $=f_{k 1} * f_{j 1}^{*}(a)$ so $\mu_{k j} \in E_{j} \cap F_{k}$. It follows from (4.5) that the functions $\mu_{k j}$ have the properties stated in the lemma.

For the rest of the paper $G=S U(2)$ and (S) is the Lie algebra of $G$. Let $Q$ be the projection onto the space of class functions in $L^{2}(G)$. Then for any continuous function $g$ on $G, Q g$ is the continuous class function on $G$ defined by

$$
Q g(a)=\int_{G} g\left(b a b^{-1}\right) d b .
$$

If $\mu_{i j}$ is a coordinate function of an irreducible representation of $G$ with character $\chi_{n}$, then using the relations in $[5$, p. 73] we can show that

$$
Q \mu_{i j}=\delta_{i j} \chi_{n} / n \text {. }
$$

For any $D \in \mathscr{S}$ and any $T \in[0, \pi]$ the map $\mu_{T D}$ of $C(G) \rightarrow C$ defined by

$$
\mu_{T D}: g \rightarrow\left(Q\left(g D_{\chi_{2}}\right)\right)\left(x_{T}\right),
$$

where $x_{T}=\operatorname{diag}\left(e^{i T}, e^{-i T}\right)$ is easily seen to be a measure on $G$.

Proposition 4.8. Let $t \in[-2,2]$, let $\lambda_{t}$ be the function on $G$ defined by (3.31), and let $D \in$ Ss. Then

$$
D \lambda_{t}=(1 / \pi)(\sin T) \mu_{T D}, \quad T=\arccos \left(\frac{1}{2} t\right)
$$

where $\mu_{T D}$ is defined in (4.7), i.e.,

$$
\pi\left(D g, \lambda_{t}\right)=-(\sin T)\left(Q\left(g D \chi_{2}\right)\right)\left(x_{T}\right)
$$

for all $g \in C^{\infty}(G)$.

Proof. The result is clear if $t= \pm 2$, so assume $t \in(-2,2)$. Let $\mathfrak{A}$ be the abelian subalgebra of (S) generated by $D$, and for each positive integer $n$ let $a \rightarrow\left(\mu_{k j}^{n}(a)\right)$ be an irreducible $n$-dimensional matrix representation of $G$ and let $\phi_{1}^{n}, \ldots, \phi_{n}^{n}$ be 
real linear functionals on $\mathfrak{A}$ having the properties described in (4.2)-(4.4). To prove (4.10) it is sufficient to show that

$$
\pi i \phi_{j}^{n}(D)\left(\mu_{k j}^{n}, \lambda_{t}\right)=-(\sin T)\left(Q\left(\mu_{k j}^{n} D \chi_{2}\right)\right)\left(x_{T}\right)
$$

for $1 \leqq n<\infty$ and $1 \leqq k, j \leqq n$. For $n>1$ we obtain from (4.6), (3.28), and (3.32)

$$
\begin{aligned}
\left(\mu_{k j}^{n}, \lambda_{t}\right) & =\left(\mu_{k j}^{n}, Q \lambda_{t}\right)=\left(Q \mu_{k j}^{n}, \lambda_{t}\right)=\delta_{k j}\left(\chi_{n}, \lambda_{t}\right) / n \\
& =\delta_{k j}((\sin (n-1) T / \pi n(n-1))-(\sin (n+1) T / \pi n(n+1))),
\end{aligned}
$$

and hence for all $n>1$

(4.12) $\pi n i \phi_{j}^{n}(D)\left(\mu_{k j}^{n}, \lambda_{t}\right)=i \delta_{k j} \phi_{j}^{n}(D) \sin T\left(\left(\chi_{n-1}\left(x_{T}\right) /(n-1)\right)-\left(\chi_{n+1}\left(x_{T}\right) /(n+1)\right)\right)$.

This equation also holds for $n=1$ if we agree to set $\chi_{n-1} /(n-1)=0$ when $n=1$. Hence we will have (4.11) if we show that

$$
n Q\left(\mu_{k j}^{n} D \chi_{2}\right)=i \delta_{k j} \phi_{j}^{n}(D)\left(\chi_{n+1} /(n+1)-\chi_{n-1} /(n-1)\right) .
$$

We prove (4.13) by showing that both sides of the equation have the same inner product with $\mu_{s r}^{m}$ for $1 \leqq m<\infty$ and $1 \leqq s, r \leqq m$. By the usual orthogonality relations for coordinate functions we get

$$
\begin{aligned}
\left(i \delta_{k j} \phi_{j}^{n}(D)\left(\chi_{n+1} /(n+1)-\chi_{n-1} /(n-1)\right), \mu_{s r}^{m}\right) \\
=i \delta_{k j} \delta_{s r} \phi_{j}^{n}(D)\left(\delta_{n+1, m}-\delta_{n-1, m}\right) / m^{2}
\end{aligned}
$$

Now use (3.4) to get

$$
\begin{aligned}
\left(n Q\left(\mu_{k j}^{n} D \chi_{2}\right), \mu_{s r}^{m}\right) & =n\left(\mu_{k j}^{n} D \chi_{2}, Q \mu_{s r}^{m}\right)=n \delta_{s r}\left(\mu_{k j}^{n}, D \chi_{2} \cdot \chi_{m}\right) / m \\
& =n \delta_{s r}\left(\mu_{k j}^{n}, D\left(\chi_{m+1}-\chi_{m-1}\right)\right) / m^{2} \\
& =n \delta_{s r}\left(D \mu_{k j}^{n}, \chi_{m-1}-\chi_{m+1}\right) / m^{2} \\
& =n \delta_{s r}\left(i \phi_{j}^{n}(D) \mu_{k j}^{n}, \chi_{m-1}-\chi_{m+1}\right) / m^{2} \\
& =i \delta_{s r} \delta_{k j} \phi_{j}^{n}(D)\left(\delta_{n, m-1}-\delta_{n, m+1}\right) / m^{2} .
\end{aligned}
$$

Compare (4.14) and (4.15) and see that we have proved (4.13) which completes the proof of Proposition 4.8.

\section{BIBLIOGRAPHY}

1. S. Helgason, Differential geometry and symmetric spaces, Academic Press, New York, 1962.

2. L. Loomis, An introduction to abstract harmonic analysis, Van Nostrand, New York, 1953.

3. R. Mayer, Summation of Fourier series on compact groups, Amer. J. Math. 89 (1967), 661-692.

4. I. Segal, The two sided representation of a unimodular locally compact group, Ann. of Math. (2) 51 (1950), 293-298.

5. A. Weil, L'intégration dans les groupes topologiques, Actualités Sci. Indust., No. 1145, Hermann, Paris, 1951.

6. H. Weyl, The theory of groups and quantum mechanics, Dover, New York, 1950.

HARVARD UNIVERSITY,

Cambridge, Massachusetts 